\title{
Astrophysical Problems Involving Carbon Re-appraised
}

\author{
$\mathrm{J}$ P Hare and $\mathrm{H}$ W Kroto
}

School of Chemistry and Molecular sciences University of Sussex, Brighton, ENI $9 Q J$ UK

\section{ABSTRACT}

The molecule $c_{60}$, Buckminsterfullerene, was discovered ${ }^{1-3}$ during laboratory experiments motivated by problems associated with processes involving carbon in stars and space 4,5 . Astronomical puzzles also lay behind the experiments which led to the molecule's extraction and structure confirmation ${ }^{6-8}$. Although the resulting breakthrough has opened up exciting new avenues of chemistry, physics and materials science here on earth' the original astrophysical questions still remain and are even more tantalising now than they were before. Some of the puzzles are here re-addressed in the light of the new understanding which the fullerene discovery has brought. Indeed we shall look at the questions through magenta coloured spectacles and note that there are new and even more intriguing parallels between the behaviour of carbon on earth and space. The article contains a brief account of the processes responsible for the synthesis of carbon in stars and its dissemination throughout the Galaxy as this information is deemed necessary to gain an intrinsic understanding of the amazing role carbon plays in nature.

\section{INTRODUCTION}

There are several problems involving carbon where some links between $\mathrm{C}_{60}$, chains and dust are possible $2,10,11$, perhaps even likely, and it is useful to re-appraise some of the leading questions in the light of our new understanding. The aim here is to understand these questions better in the hope that answers will soon be forthcoming. Fullerene-60 analogues are only likely to be important in space if the geodesic cage structure (sigma electron network) remains essentially intact and that the integrity of the fullerene pi-electron system is disrupted as little as possible. Otherwise a vast range of analogues appears feasible and/or cage fragmentation would be expected to take place. Secondly, as fullerenes are very photostable in cold beams ${ }^{12}$ and exhibit a peculiar instability in some condensed phases ${ }^{13}$ they may be more stable in space than on earth. The detailed arguments which suggest that fullerene analogues may be the missing links in the Galactic Carbon Chain (of events) have been discussed by Kroto and Jura 14

\section{The Diffuse Interstellar Bands (DIBs).}

The identity of the carrier of the DIBs (a set of some 80 electronic absorption features which are too broad to be atomic lines) has been a major puzzle ever since they were identified as interstellar features in 1936. Their properties have been summarised by Herbig 15,16 . Numerous suggestions have been made as to the nature of the carrier and at present the consensus lies between some moderately large "stable" molecule and grain-like material in which atoms are trapped giving rise to matrix-like spectra. The problem is that this material appears to be is ubiquitously abundant even in 
the hostile environment of space and yet, astoundingly, has never been identified on earth. Recently Sarrelt and Fossey 18 have shown convincingly that five DIB features occur in emission from the "Red Rectangle" a carbon-rich star, HD44179, which exhibits a bipolar outflow with a roughly rectangular emission pattern. These features are sitting on top of a very broad intense red continuum emission ${ }^{19}$ which Dyley has suggested is from some form of $\mathrm{H} / \mathrm{C}$ containing material 20 . $\mathrm{C}_{60}$ or an analogue was suggested as a possjble carrier when the molecule was first found to form spontaneously. There are intriguing parallels between fullerene-60 and the DIB carrier which have prompted the conjecture to be probed further $2,10,11,14,21$. The ubiquity of $<13.6 \mathrm{eV}$ radiation indicates that if $\mathrm{C}_{60}$ is formed in space (and is as stable as beam experiments suggest) it will be ionised. The case for ionic analogues 10,11 has been discussed 21 . The discovery of endohedral fullerene complexes in which the atom is inside the cage such as (La) has prompted interest in the astrophysical importance of such species and several calculations have been carried out on their spectra23-26. Perhaps the most interesting analogues are however exohedral complexes and the possibility that charge transfer bands associated with such species as ()$^{+}$where $M$ is an abundant interstellar species such as $\mathrm{Na}, K$, $\mathrm{Ca}, \mathrm{Fe}, \mathrm{S}, \mathrm{O}$ etc has been discussed in detail ${ }^{14}$. These ideas are supported by the recent experimental results of Huang and Freiser 27 .

The Unidentified 2170 A Band.

Another feature which has also been the subject of numerous studies is a strong absorption centered at ca $2170 \mathrm{~A}^{28-30}$. This feature is very strong and in general always observed at 2170A. stecher and Donn suggested this was caused by carbon when they first detected the feature 29 . The line shape is also relatively constant although there are one or two objects in which the feature appears to shift slightly 30 Interestingly Day and Huffman 31 have studied the scattering from pure small carbon particle and finds a feature centered near $2400 \mathrm{~A}$. It has been noted ${ }^{14}$ that in the highly $\mathrm{H}-$ depleted RCorBor stars the feature appears to shift considerably to ca $2400 \mathrm{~A}^{30}$. Protonated species are common in the ISM and thus the possibility that a protonated exohedral fullerene complex $(+) \mathrm{H}$ should be seriously considered ${ }^{14}$. Such a species is expected to have a very strong, very broad, charge-transfer electronic absorption near $6 \mathrm{eV}$ (ca $2100 \mathrm{~A}$ ) 14 which is quite close to $2170 \mathrm{~A}$, so a contribution to the interstellar extinction from such a transition may be important.

\section{The Unidentified Infrared Bands (UIBs).}

The development of sensitive IR telescopes has led to the detection of IR emission from circumstellar material ${ }^{32}$. Comparisons between some discrete circumstellar features with laboratory IR measurements of known polycyclic aromatic hydrocarbons (PAHs) are sufficiently good that the assignment to PAH-like carriers is quite convincing $3-35$. The $\mathrm{C}_{60}$ discovery suggests that hydrogenated curved/partly-closing structures might also occur and such objects have been shown to yield certain IR features consistent with observation ${ }^{36}$.

There are of course a multitude of possible species which can form from some complex mix of small compounds to large aggregates of 
organic material such as soots and tars. It has been suggested 34 that the interstellair radiation flux which pumps the observed bands is such that the pattern of emission frequencies detected can be used to set a bound on the size of the emitting species as containing 20-40 atoms. This calculation however depends on the assumption that on absorption of a photon the energy is uniformly distributed and the species or grain emits IR radiation with a characteristic thermalised profile. It is not however clear that localised chromophore excitation cannot occur due to inefficient energy transfer in heterogeneous aggregates. Intramolecular vibrational relaxation and the relationship with radiative processes are poorly understood even for small molecules and even less so in complex, loosely bound aggregates.

of course hydrogen is present in copious quantities in moşt astrophysical objectts, however the observations of Gerhardt et al 37 and Howard et al ${ }^{38}$ have shown polyynes are a prelude to soot formation and that $\mathrm{C}_{60}$ forms in a sooting flame and can be extracted. There is thus every prospect that fullerenes form wherever polyynes and dust form in space, even in the presence of hydrogen and oxygen. There is still some way to go before the question of how important the role of non-planar carbonaceous networks is during soot formation, however it should be investigated as soot formation appears to be so poorly understood ${ }^{39}$.

\section{Interstellar and Circumstellar Dust}

The dust in space is a most fascinating material, playing key roles in many important astrophysical processes ${ }^{28}$. For instance it appears that grain surface catalysis is the only way to explain the formation of $\mathrm{H}_{2}$ in space ${ }^{40}$. For the purposes of this discussion we note that the dust in the dark clouds protects molecules from dissociation by starlight and so enables the heat generated on gravitational collapse to be dissipated by low temperature rotational emission by $c O$ and so allow later phases of cloud collapse to occur - ultimately to form new stars. Dust certainly forms in the shells of red giant stars and also around novae, supernovae, and planetary nebulae and the latter are proving to be rather interesting sources of carbon. The planetary AFGL 2688 is a hot star surrounded by a donut-shaped dense molecular gas and dust cloud 41,42 . The star sits in the hole in the middle of the donut and emanating from the star, along the axis of the donut, is a bipolar outflow of high velocity gas $-\mathrm{H}$ and $\mathrm{CO}$ at ca $50 \mathrm{~km} \mathrm{~s}^{-1}$. The visible emission contour has caused it to be called the "Egg Nebula"41. Rieu et al 42 discovered a curious feature about this star: The donut contained the molecules that the ion-molecule theories 434 readily explain (ammonia etc) but far out from the center, the conical bipolar outflow is surrounded by hollow cone of gas, rich in polyynes ${ }^{42}$. It has been suggested that these chains are produced by grain fragmentation due to grain-grain collisions 45 . Carbon cluster beam photofragmentation studies have shown that macroscopic carbon clusters can break down to such chains and $\mathrm{C}_{60}$ also appears ${ }^{12}$. Thus laboratory experiments suggest that $c_{60}$ analogues may also occur in such regions ${ }^{2}$.

Studies of the polarisation of scattered light by interstellar dust suggests that it in some regions it may consist of elongated particles ${ }^{28}$. The work of Iijima 46 and Endo 47 indicates how such 
elongated carbon miçrofibres may grow under conditions in which $\mathrm{C}_{60}$ forms. Microfibres with diameters of 30-100A with graphite walls containing from 2-50 layers of graphite have now been detected. These structures are closely related to the structures observed earlier by Iijima ${ }^{48}$. On the basis of our new understanding of closed carbon giant networks 49,2 a possible growth mechanism for such microfibers has been proposed ${ }^{0}$. The possibility that such tiny tubular graphite structures (Zeppelenes?) are feasible adds a new dimension to the possible answers to a range of fascinating spectroscopic astronomical observations such as those by sellgren who observed IR emission consistent with microscopic particles of these dimensions ${ }^{51}$. Wright has suggested that spherojial graphite structure cannot account for the observed emission ${ }^{2}$. It should however be noted that attention has been drawn to the fact that the apparent epitaxial control of growth in general breaks down and in later stages of accretion from the gas phase, graphite layers beçome more chaotic and so-called "amorphous" texture appears to develop 53 .

\section{Meteoritic Carbon}

Most interesting observations have been made by Lewis et al $1^{54}$ who have detected diamond domains in meteorites. These inclusions are most interesting and how they might arise is food for thought. One suggestion is that they might form by metamorphosis, initiated perhaps by shock waves, of the internal structure of quasi icosahedral concentric shell graphite dust included in the meteorite 55 .

One of the most intriguing aspectss of the meteorites are their curious isotope anomalies. Clayton 56 has suggested that the existence of almost pure ${ }^{22} \mathrm{Ne}$ in some carbonaceous chondrites might be explained if it is a remanant of $22 \mathrm{Na}$ formed in supernovae. He suggests that the $22 \mathrm{Na}$ (which has a $2.5 \mathrm{yr}$ half-life) is ejected from the star and co-condenses with carbon in the surrounding dust shell. The detection of endohedral metal complexes such as (M) where $M=L a$ etc 22,27 and the elegant recent observation of He encapsulation during collisions with fullerene -60 by Weiske et al ${ }^{57}$ suggest that the endohedral fullerene complexes might well play a role in these well known isotope anomalies involving carbon. Soon after the discovery of $\mathrm{C}_{60}$, Heymann ${ }^{58}$ considered the (He) complex from an astrophysical viewpoint.

\section{Conclusions}

Several astrophysical problems have been described which involve carbon chains and dust and attention has been drawn to various chemical scenarios in the laboratory which yield very similar conditions. The cluster beam studies indicate that chains (or their monocyclic ring analogues) form first which then evolve into fullerenes and carbon microparticles in some yet to be fully understood way. The fascinating observation of Rubin et al 59 that $\mathrm{C}_{30}$ monocylic rings dimerise spontaneously to $\mathrm{C}_{60}$ can be rationalised by a scheme in which a concerted series of cycloaddition steps can result in a graphitic/fullerene network 6 . Furthermore recent evidence suggests that fullerenes may even be the direct precursors of pure carbon microparticle accreting carbon directly from the vapor ${ }^{50}$. On the basis of a wealth of circumstantial evidence we have argued fullerene analogues must have 
an important role in the "Galactic carbon cycle". It has been pointed ${ }^{14}$ out that fullerene analogues do have several properties that justify their carful consideration as carriers of some ubiquitous astrophysical features and that if they are not responsible there is some other as yet unidentified mystery involving carbon to be unraveled ${ }^{14}$.

Acknowledgements We are grateful to Simon Balm, Laurence Dunne, Mike Jura, Sydney Leach, Peter Sarre and Jim watson for valuable discussions. We thank the Royal Society, British Gas and SERC.

\section{REFERENCES}

1) Kroto, H. W.; Heath, J. R.; O'Brien, S. C.; C url, R. F.; Smalley, R. E., Nature (London) 1985, 318, 162-163.

2) Kroto, H. W. Science 1988, 242, 1139-1145.

3) Curl, R. F. and Smalley, R. E. Science 1988, 242, 1017-1022.

4) Heath, J. R. ; Zhang, Q.; O'Brien, S. C.; Curl, R. F.; Kroto, H. W. ; Smalley, R. E. J. Am. Chem. Soc. 1987, 109, 359-363.

5) Kroto, H. W.; Heath, J. R.; O'Brien, S. C; Curl, R. F. ; Smalley, R. E. Astrophys. J. 1987, 314, 352-355.

6) Kraetschmer, W.; Lamb, L. D. ; Fostiropoulos, K.; Huffman, D. R. Nature (London). 1990, 347, 354-358.

7) Taylor, R.; Hare, J. P.; Abdul-Sada, A. K.; Kroto, H. W. J. Chem. Soc. Chem. Commun. 1990, 1423-1425.

8) Kroto, H. W., Angewandte Chemie 1990, in press

9) Kroto, H. W., Allaf, A. W.; Balm, S. P., Chem. Revs. 1991, 91, 1213

10) Kroto, H. W. Polycyclic Aromatic Hyrocarbons and Astrophysics, Leger, A.; d'Hendecourt, L. B. eds.; Reidel: Dordrecht, 1987, pp. 197-206.

11) Kroto, H. W. Ann. Phys. Fr. 1989, 14, 169-179.

12) Heath, J. R.; O'Brien, S. C.; Curl, R. F.; Kroto, H. W. ; Smalley, R.E. Comments. Condens. Matter Phys. 1987, 13, 119-141

13) Taylor, R.; Parsons, J. P.; Avent, A. G.; Rannard, S. P.; Dennis, T. J.; Hare, J. P.; Kroto, H. W.; Walton, D. R. M., Nature 1991, 351, 277.

14) Kroto, H. W.; Jura, M.; in press.

15) Herbig, G. H. Astrophys. J. 1975, 196, 129-160.

16) Herbig, G. H. Astrophys. J. 1988, 331, 999-1003.

17) Sarre, P. J. Nature, 1991, 351, 356.

18) Fossey, S. J. Nature, 1991, 353, 393.

19) Cohen, M. et al., Astrophys. J. 1975, 197, 179.

20) Duley, w. W., Mon. Not. R. astr. Soc. 1985, 215, 259-263.

21) Leger, A.; d'Hendecourt, L.; Verstraete, L.; Schmidt, w. Astron. Astrophys. 1988, 203, 145-148.

22) Heath, J. R. ; O'Brien, S. C.; Zhang, Q.; Liu, Y.; Curl, R. F.; Kroto, H. W.; Smalley, R. E. J. Am. Chem. Soc. 1985, 107, 77797780 .

23) Ballester, J. L.; Antoniewicz, P. R.; Smoluchowski, R. Astrophys. J. $1990,356,507-512$.

24) Cioslowski, J.; Fleischmann, E. D., J. Chem. Phys. 1991, 94, 3730 .

25) Chang, A. H. H.; Ermler, W. C.; Pitzer, R. M., J. Chem. Phys. $1991,94,5004$.

26) Wastberg, B.;Rosen, A., Physica Scripta. 1991, 44, 276-288.

27) Huang, Y.; Freiser, B. S., Nature 1991, $* \star * \star *$ 
28) Savage, B. D.;. Mathis, J. S., Ann. Rev. Astrophys. 1979, 17, 73-111.

29) Stecher, T. P.; Donn, B., Astrophys. J. 1965, 142, 1683.

30) Fitzpatrick, E. L.; Massa, D., Astrophys. J. 1986, 307, 286.

31) Day, K. L.; Huffman, D. R., Nature Physical Science, 1973, 243, 50-51.

32) Holm, A. V.; Wu, C.; Doherty, L. R., Astro. Soc. Pac. 1982, 94, 548-552.

33) Duley, W. W.; Williams, D. A. Mon. Not. R. Astron. Soc. 1988, 231, 969-975.

34) Leger, A.; Puget, L. J. Astron. Astrophys. 1984, 137, L5-L8.

35) Allamandola, L. J.; Tielens, A. G. G. M.; Barker, J. R. Astrophys. J. 1985, 290, L25-L28.

36) Balm, S.P.; Kroto, H. W. Mon. Not. R. Astron. Soc., 1990, 245, 193-197.

37) Gerhardt, P.; Homann, K. H. J. Phys. Chem. 1990, 94, 5381-5391.

38) Howard, J. B.; McKinnon, J. T.; Makarovsky, Y.; Lafleur, A. L.; Johnson, M. E., Nature 1991, 352, 139-141.

39) Harris, S. J.; Weiner, A. M., Ann. Rev. Phys. Chem. 1985, 36, 31-52.

40) Mccrea, W. H.; McNally, D., Mon. Not. Roy. Astronom. Soc. $1960,121,238$.

41) Ney, E. P., Sky and Telescope 1975, Jan, 21.

42) Nquyen-Q-Rieu; Winnberg, A.; Bujarrabal, V., Astron. Astrophysics. 1986, 165, 204-210.

43) Herbst, E.; Klemperer, W. Astrophys. J. 1973, 185, 505-533.

$44)$ Dalgarno, A.; Black, J. H. Rep. Prog. Phys. 1976, 39, 573-612.

45) Jura, M.; Kroto, H. W., Astrophys. J. 1990, 351, 222-229.

46) Iijima, S., Nature 1991, 354, 57.

47) Endo, M., personal communication.

48) Iijima, S. J. Cryst. Growth, 1980, 5, 675-683.

49) Kroto, H. W.; MCKay, K. G. Nature (London), 1988, 331, 328-331.

50) Endo, M. and Kroto, H. W., to be published.

51) Sellgren, K., Astrophys. J. 1984, 277, 623-633.

52) Wright, E. L. Nature (London) 1988, 336, 227-228

53) Kroto, H. W., Iijima, S., to be published

54) Lewis, R. S.; Ming, T.; Wacker, J. F.; Anders, E.; Steel, E., Nature $1987,326,160$.

55) McKay, K. G.; Dunne, L. J.; Kroto, H. W.; in preparation.

56) Clayton, D. D. Nature (London) $1975,257,36-37$.

57) Weiske, T.; Bohme, D. K.; Hrusak, J; Kratschmer, W; Schwarz, Angew. Chem. Int. Ed. Engl. 1991, 30, 884 .

58) Heymann, D. J. Geophys. Res. B 1986, 91, E135-138.

59) Rubin, Y.; Kahr, M.; Knobler, C. B.; Diederich, F.; Wilkins, C. L., J. Am. Chem. Soc. 1991, 113, 495-500.

60) Kroto, H. W.; Walton, D. R. M.; Post-Fullerene Organic Chemistry in Chemistry of Three-Dimensional Polycyclic Molecules, Editors; Osawa, E. and Yonemitsu, $0 ., \mathrm{VCH}$ International: New York. 


\section{GREENBERG}

a) What is the visual absorption by $\mathrm{C}_{60}$ as a function of wavelength.

b) Could it contribute to the observed extinction.

c) How much $C$ is needed to produce visual extniction if in $\mathrm{C}_{60}$

KROTO

$a+b)$ The $c_{60}$ spectrum is now known and it is not ovious that it contributes to any optical features. The visible absorption is very weak. The bands at 2100 and $2500 \mathrm{~A}$ are very strong. However in the regions where it is likely to be seen (visibly) it is also likely to be ionised so $\mathrm{C}_{60}{ }^{+}$is probably the dominant analogue. Also in any region where $\mathrm{HCO}^{+}$is abundant the most likely species is $\mathrm{C}_{60} \mathrm{H}^{+}$.

c) Visible extinction of the neutral $\mathrm{c}_{60}$ molecule is fairly weak as there are only forbidden transitions in this region

\section{GREENBERG}

If DIBs are due to $K$ then there should be an anticorrelation between $K$ absorption and the DIBs. (Incidentally, I once - 30 years ago - questioned the $\mathrm{Ca}$ line anticorrelation with the $4430 \mathrm{~A}$ band to indicate $\mathrm{Ca}$ as a possible source of DIB carrier). Maybe $\mathrm{Ca}$ is a better choice for your mechansim.

\section{KROTO}

Our paper (Kroto and Jura) suggests that the DIB carriers might be $\mathrm{C}_{60} \mathrm{M}$ ionised complexes where $\mathrm{M}$ is an alkali metal or any abundant atom. $\mathrm{Ca}, \mathrm{Na} \mathrm{K}$ and $\mathrm{Mg}$ as well as 0 and $S$ etc are all excellent candidates for the attached species. The $\mathrm{Ca}$ and $\mathrm{K}$ anticorrelation are possibly interesting support for the proposal and in fact we have discussed $\mathrm{Ca}$ in our paper. In my verbal presentation $I$ discussed $K$ as an example of the type of atom likely to give rise to charge transfer bands. (doubly charged species such as $\mathrm{C}_{60}{ }^{-\mathrm{Ca}^{++}}$are also very interesting species).

\section{GREENBERG}

If DIBs are due to $K$ in/on $C_{60}$ do you need to give the DIB strength. Could we be running into a cosmic abundance problem since $\mathrm{N}_{\mathrm{C}} / \mathrm{N}_{\mathrm{Ca}}$ is large per DIB molecule.

KROTO

Under certain conditions in the laboratory the amount of carbon in $\mathrm{C}_{60}$ and other fullerenes to carbon in soot varies from 10-20\%. In benzene combustion flames it can be as high as ca $7 \%$ of the soot collected. The charge 
transfer bands associated with exohedral coplexes of the kind discussed are associated with electron jumps of the order of 6-7A which are more than an order of magnitude larger than for standard atomic and molecular electronic transitions resulting in bands some 50-100 times stronger than usual fully allowed transitions.

\section{WILLIAMS} the lab?

What is the C:H ratio in the experiments you do in

\section{KROTO}

This is a difficult question to answer as the process we observe is highly inhomogeneous. A pure carbon plasma is vaporised from graphite surface by a laser into a $\mathrm{He} / \mathrm{H}_{2}$ atmosphere. As the hot plasma expands the contact surface causes complex reactions between carbon atoms (which are themselves clustering) at the interface and reacting with $C$ and $C / H$ radicals. Even in pulses with $\mathrm{He} / \mathrm{H}_{2}$ ratio of $\mathrm{ca} 1: 1$ (by volume) we still see $\mathrm{C}_{60}$ formation. Even more remarkable is the observation by ulf Sassenberg and Bosse Lindgren in Stockholm who have seen $\mathrm{C}_{60}$ formation in pure oxygen gas pulses! Also, Howard and his co-workers at MIT have seen some $7 \%$ of fullerene material in soot from a benzene flame. To sum up, $c_{60}$ is detected whenever carbon particles are produced in the gas phase.

\section{L d'HENDECOURT}

The production of $c_{60}$ by Krätschmer (as well as others) yields large amounts of $\mathrm{C}_{60}$ in a very specific way (plasma of carbon in He at "high" pressure). Are such experiments applicable to the atmospheres of (carbon) stars where hydrogen dominates and the expected pressures are orders of magnitude smaller?

KROTO

I feel that the key factor is whether the conditions favor carbonaceous dust production. Whenever we see carbon particles in the laboratory we also invariably now see $\mathrm{C}_{60}$. Intuition certainly suggests that the higher the $\mathrm{C} / \mathrm{H}$ ratio is, the higher the $\mathrm{C}_{60} /$ soot ratio is likely to be. When $\mathrm{H} / \mathrm{C}=0$ we know $\mathrm{C}_{60}$ can make up some $15 \pm 5 \%$ of the soot and RCorBor and similar stars are clearly ideal objects. The recent combustion data suggest that even in high $(\mathrm{H}+\mathrm{O}) / \mathrm{C}$ regions the $\mathrm{C}_{60}$ to dust ratio may be between $1-10 \%$ 